\title{
SURGICAL UNCOVERING AND STIMULATION OF PHYSIOLOGICAL ERUPTION OF PALATALLY IMPACTED MAXILLARY CANINES: CASE REPORTS
}

\author{
Miroslava Yordanova $^{1}$, Svetlana Yordanova ${ }^{1}$, B. Vladimirov ${ }^{2}$ \\ ${ }^{1}$ Department of Orthodontics, \\ ${ }^{2}$ Department of Maxillofacial Surgery, \\ Faculty of Dental Medicine, Medical University - Plovdiv, Bulgaria
}

\begin{abstract}
Introduction. The orthodontist treating patients with palatally impacted canines usually strives to build a complex treatment plan, based on uncomplicated and biological orthodontic and surgical techniques.

The aim of the present report was to demonstrate by case reports the potential of the open surgical technique for stimulation of physiological eruption of palatally impacted canines.

Material and methods. Three patients aged 13 to 20 years with various degrees of unilateral and bilateral palatal impaction of maxillary canines were managed by the described technique.

Results and discussion. All canines underwent autonomous eruption after all soft- and hard-tissue obstructions on their paths were removed.

Conclusion. The described method of surgical uncovering and autonomous eruption created conditions for biological eruption of the palatally impacted canines into the oral cavity and facilitated considerably the subsequent orthodontic treatment for their proper alignment in the dental arch.
\end{abstract}

Key words: palatally impacted canines, surgical exposure, autonomous eruption

After mandibular third molars, the maxillary canines are the second most commonly impacted teeth (1), with palatal impactions prevailing over buccal impactions $(2,3)$. Treatment approaches are aimed at the canines' correct occlusion, as well as function and esthetics of the dentition and can be divided into preventive and surgical. The preventive approach involves diagnosing the early signs of palatal displacement of permanent canines and early extraction of primary canines in order to achieve selfcorrection of the improper eruption (4). If diagnosis is belated and prevention of impaction is impossible surgicalorthodontic treatment should be performed.

Various surgical techniques for exposing palatally impacted canines exist (5): 1. technique of open eruption; 2. technique of closed eruption; 3. open eruption through a window; 4. tunnel extrusion, etc. Within the different approaches there exist two main options for the subsequent eruption of the impacted teeth (6): natural autonomous eruption and forced eruption under orthodontic traction.

The choice of a surgical approach still remains a question of personal preference for the surgeon and orthodontist because the scientific literature lacks satisfactory evidence in favor of one of the techniques $(7,8,9)$. Additional studies comparing directly the various methods and their advantages as regards the periodontal status of the impacted teeth are needed $(10,11)$.

The aim of the present report was to demonstrate the potential of the open surgical technique for stimulation of physiological eruption of palatally impacted canines by case reports.

\section{MATERIAL AND METHODS}

Three patients aged 13 to 20 years with various degrees of unilateral and bilateral palatal impaction of maxillary canines were managed by the described technique. The teeth had different degrees of impaction in relation to the vertical and horizontal planes. Surgical exposure was performed before orthodontic treatment in one of the cases, while it was done during the first stage of orthodontic treatment in the rest. Standard procedure for surgical uncovering and autonomous eruption was followed for all teeth (12):

-incision of palatal mucosa and periosteum apically to the gingival sulcus on premolars and ipsilateral incisors, and then following the midline;

-elevation of a mucoperiosteal flap;

-removal of bone overlying the canine's crown from its tip down to the level of the cementoenamel junction by means of a curette or a handpiece and bur (in deeply situated teeth);

-curettage of the follicle at the periphery of the dental crown;

-repositioning of the flap;

-creating a small fenestration in the flap over the 
crown;

-suturing the flap;

-placement of a periodontal dressing (VOCO pac) for 7 to 10 days;

-giving standard instructions for oral hygiene and eating.

\section{Clinical case \# 1.}

The patient D.B. aged 13 years was referred by his general dentist after impacted maxillary canines were diagnosed on panoramic and occlusal radiographs. Thorough clinical, photometric, biometric and cephalometric investigations revealed persistent upper primary canines, compression and protrusion of both dental arches, deep bite, Angle's Class I, and skeletal Class II. Intraoral palpation and the occlusal radiograph (Fig. 1) helped confirm the palatal impaction of the maxillary permanent canines. The lack of teeth 18,38 , and 48 as well as the degree of impaction of the two canines were found on the panoramic X-ray (Fig. 1). They were almost horizontally positioned, the incisal tip of the left canine being at the level of apex of the left central incisor, while the right canine was located approximately at the middle third of the root of the right lateral incisor. According to Ericson and Kurol's classification (4) the medial displacement of the crown of the canine in relation to the long axis of the lateral incisor was grade III impaction for tooth \# 13 and grade $\mathrm{V}$ impaction for tooth \# 23. The close position of the canines to the roots of the incisors and premolars was considered an indication for cone-beam CT (CBCT). No root resorption was found on $\mathrm{CBCT}$ images (Fig. 1).
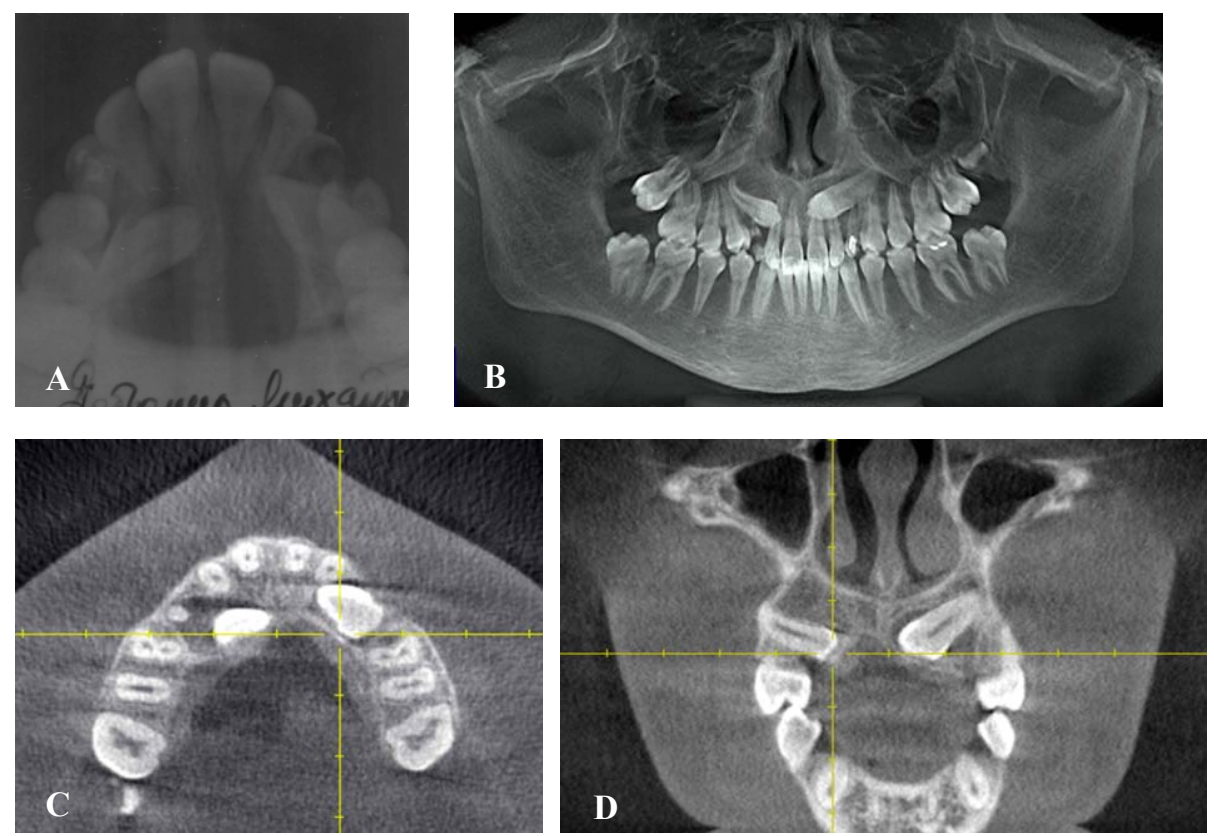

Fig. 1. Radiographs of patient D.B. aged 13 taken before surgical-orthodontic treatment (A - occlusal x-ray; B - panoramic x-ray; C and D - CBCT).

After a consultation with a maxillofacial surgeon, the possibilities for surgical exposure and orthodontic treatment and their risks, especially of failure, were discussed with the patient and the patient's family. The following treatment plan was elaborated and accepted:

Extraction of tooth \# 53; uncovering of tooth \# 13 before commencement of orthodontic treatment, then waiting for its autonomous eruption in the palate (Fig. 2 A, B, C, D).

Leveling and alignment of teeth in the upper dental arch by means of fixed appliance MBT 0.22 “.
Bonding of a bracket on tooth \# 13 and movement of the canine laterally towards the arch (Fig. 2 - E).

Extraction of tooth \# 63, uncovering of tooth \# 23, then waiting for its autonomous eruption (Fig. 2 - F, G, H, I).

Leveling and alignment of teeth in the lower dental arch by means of fixed appliance MBT 0.22 “.

Bonding of a bracket on tooth \# 23 and gradual positioning of the canine into the arch (Fig. 2 - J, K).

Correction of the deep bite and coordination of the two dental arches. 

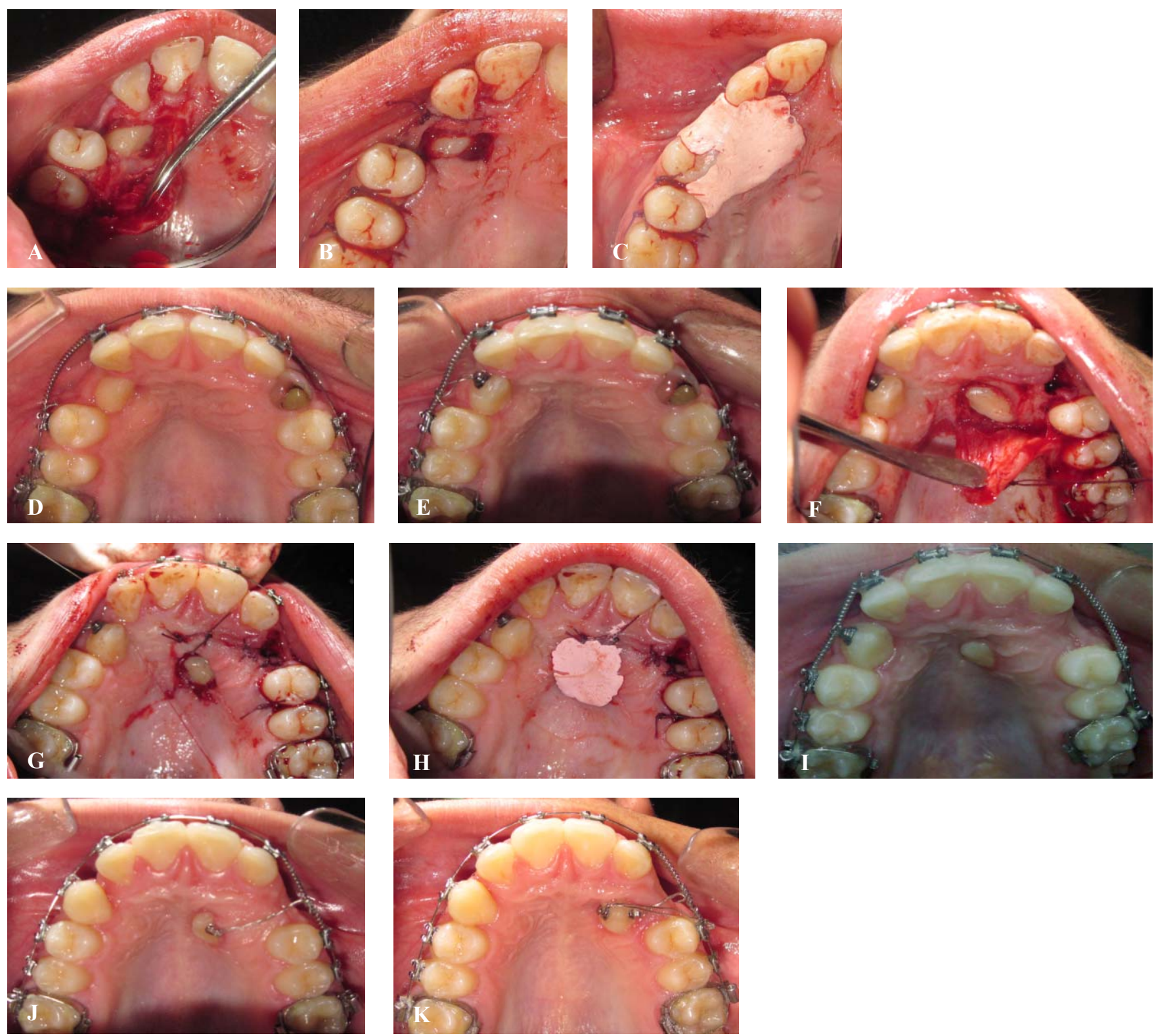

Fig. 2. Stages of treatment - patient D.B. aged 13 years.

\section{Clinical case \# 2.}

The patient D.M. aged 15 years was referred by the general dentist because of persistent teeth \# 53 and \# 63 . The complete orthodontic examination revealed the following problems: persistent upper primary canines; compressed dental arches with 2-3 $\mathrm{mm}$ of insufficient space for teeth \# 13 and \# 23; deep bite; Angle's dental Class II 1 and skeletal Class I.

Swelling of the palate behind teeth \# 12, 53, 22, 63 which was more pronounced on the left was found by palpation. The occlusal radiograph (Fig. 3) also confirmed palatal impaction for both canines. The panoramic X-ray (Fig. 3) helped to identify the dental germs of the four wisdom teeth and the degree of impaction of the upper canines. The upper right canine was inclined and vertically its tip was situated slightly above the cemento-enamel junction of tooth \# 11. Its medial position in relation to tooth \# 12 correlated to grade IV impaction. The left canine demonstrated almost normal direction of eruption and grade II medial displacement. It was positioned low and close to the palatal mucosa.

The patient was offered a treatment plan involving extraction of primary canines, uncovering of the permanent canines by the described technique and their alignment in the dental arch. 

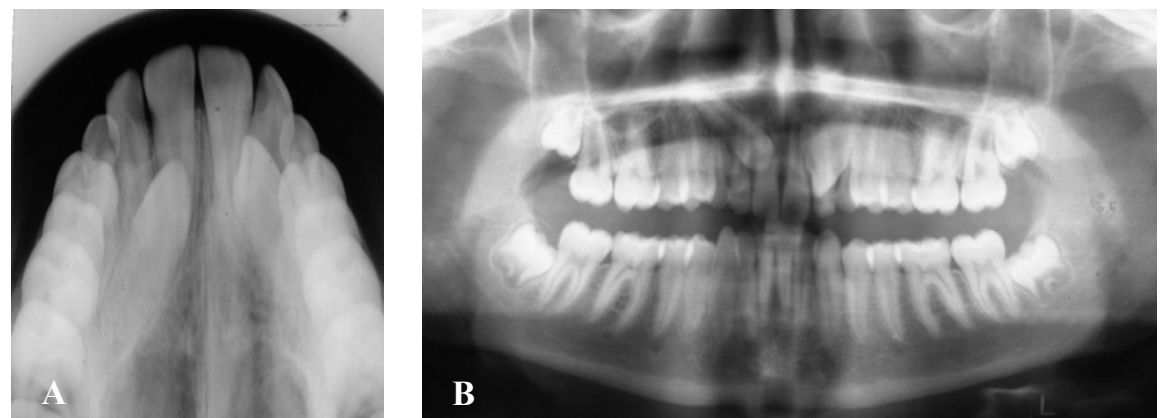

Fig. 3. Radiographs of patient D.M. aged 15 taken before surgicalorthodontic treatment (A - occlusal x-ray; B - panoramic X-ray).

Tooth \# 23 underwent autonomous eruption palatally to tooth \# 22 two months after the beginning of orthodontic treatment (Fig. 4 - A). Due to the considerable tightness of the palatal mucosa surgical gingivectomy was planned to accelerate the full eruption of the tooth (Fig. 4 - B, C). The surgical uncovering was applied on tooth \# 13 followed by its subsequent autonomous eruption (Fig. 4 - D, E, F, G). At the same time space for the impacted canines was being created by means of a fixed appliance MBT 0.22 “ (Fig. 4)
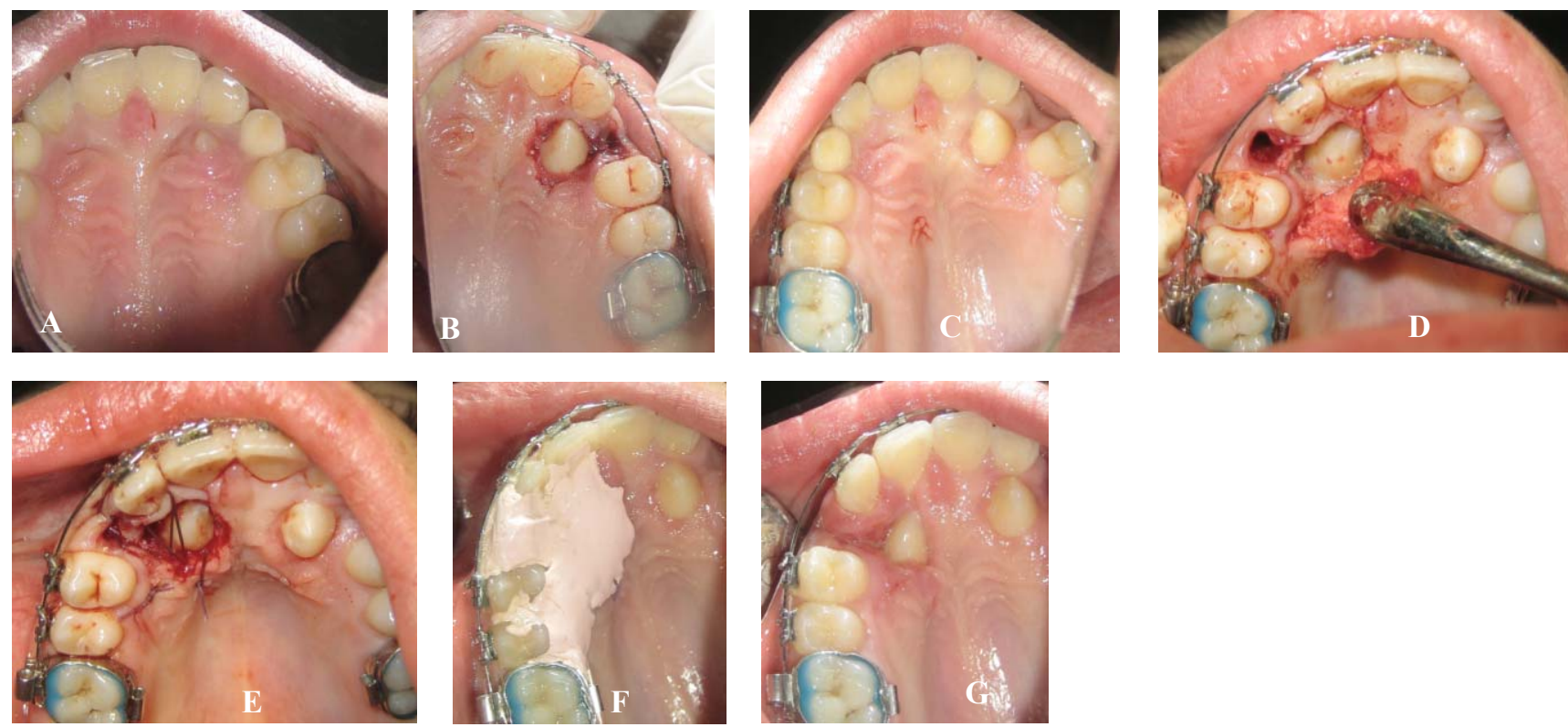

Fig. 4. Stages of treatment - patient D.M. aged 15 years

\section{Clinical case \# 3.}

The patient K.T. aged 20 years sought orthodontic help with a chief complaint of unsatisfactory smile esthetics because of lacking tooth \# 13 and irregular alignment of the other teeth. Clinical and additional orthodontic investigations found lack of tooth \# 13 and insufficient space for it in the dental arch; edge-to-edge occlusion between teeth \# 22 and \# 32, 33, as well as cross bite between \# 16, 17 and \# 46, 47; Angle's dental Class I; skeletal Class III and slight hyperdivergent type of growth. The palatal impaction of \# 13 as analyzed on panoramic radiograph (Fig. 5) was grade III of medial displacement. The crown tip of tooth \# 13 was above the cemento-enamel junction of tooth \# 11 and the canine was vertically inclined.

The surgical-orthodontic treatment plan involved: surgical uncovering of tooth \# 13 while leveling and aligning of the upper dental arch by means of a fixed appliance MBT 0.22 “ (Fig 6 - A, B); waiting for the canine's autonomous eruption and creating space in the dental arch; moving tooth \# 13 laterally and simultaneously leveling and aligning the lower dental arch (Fig. 6 - C). 


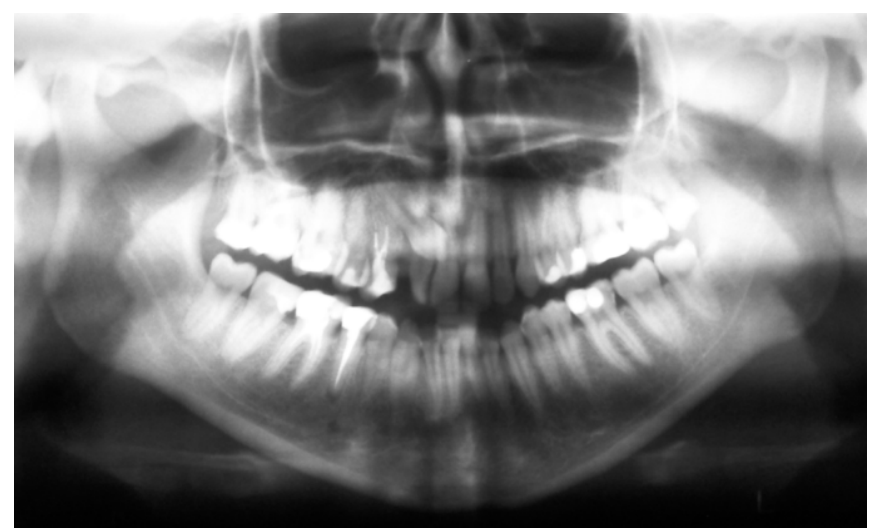

Fig. 5. Panoramic x-ray - patient K.T. aged 20 years.
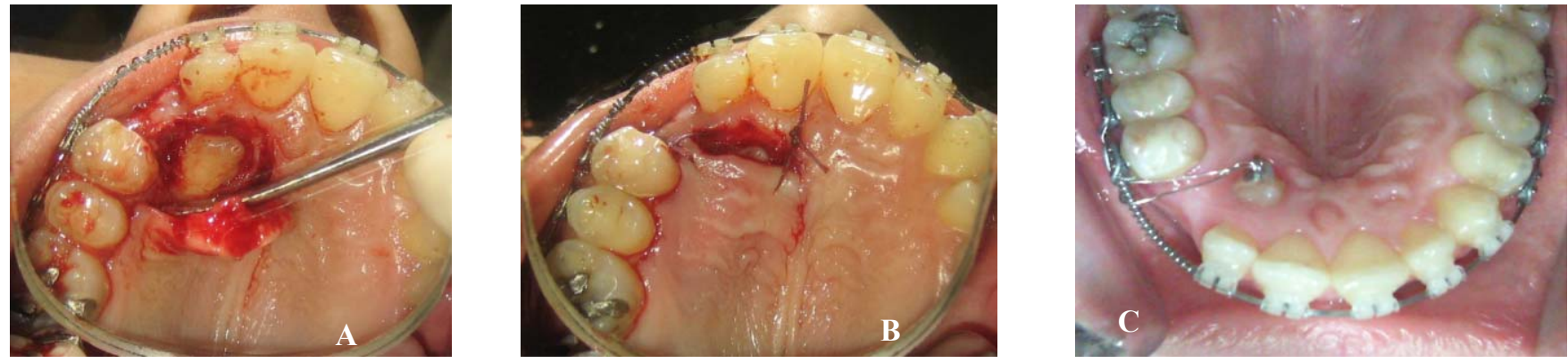

Fig. 6. Stages of treatment - patient K.T. aged 20 years.

\section{RESULTS}

Autonomous eruption of the impacted canines after surgical uncovering was witnessed in all patients without the need for application of a vertical orthodontic force for their extrusion. The period of waiting for the autonomous eruption of the impacted canines before beginning of lateral movement towards the dental arch was as follows:

Case 1 - for tooth \# 13 - 6 months; for tooth \# 23 - 5 months.

Case 2 - for tooth \# 13 - 7 months; for tooth \# 23 - 2 months.

Case 3 - for tooth \# 13 - 5 months.

In case 1 the total time from surgical uncovering to final alignment of tooth \# 13 in the dental arch was 10 months. In case 2 tooth \# 23 was brought to its final position for a period of 11 months. The rest of the canines are still at the stage of lateral movement towards the dental arch.

\section{DISCUSSION}

Different surgical techniques find support or criticism in numerous investigations (5). The present report addressed only one aspect of the overall treatment of impacted canines - the eruption of the teeth after their surgical exposure. This important first phase is frequently associated with complications such as devitalization of teeth, loss of marginal bone, gingival recessions, trauma to neighbouring tissues, etc. That is why the clinician should strive for a treatment approach to impacted canines that is protective of the dentition and periodontium (13).

The method for exposure of palatally impacted canines used in the present study allows autonomous eruption of the teeth, which is considered a physiological type of extrusion. This technique facilitates considerably the whole orthodontic treatment. No mechanical devices for vertical extrusion of the canines need to be applied and therefore there is no need for a complicated clinical judgement of the magnitude and direction of the orthodontic force. Besides, the risks that the debonding of traction devices carries are eliminated. The orthodontist does not usually wait for the teeth to erupt below the occlusal plane, which obviates the need for disarticulation of occlusion in order to pull the crown from its palatal position (6).

Self-correction of the long axis of the canines was observed during their autonomous eruption. Some of the teeth were almost horizontally positioned but erupted for less than 6 - 7 months. Many authors prove that impacted teeth retain their ability to erupt even after the formation of the root apex (14). Such was the case with the patient K.T. aged 20 years, whose impacted canine showed autonomous eruption similar to the other teeth with unfinished formation. However, in older patients (above 30 years) and complex canine impactions Kokich (6) recommends the technique of 
open extrusion through a window in order to shorten the treatment time.

In the present case series no patient reported pain, considerable discomfort or very poor oral hygiene.

\section{CONCLUSION}

Impacted teeth can erupt spontaneously if soft- and hard-tissue obstacles on their path are eliminated. The results of the use of surgical uncovering and autonomous eruption, demonstrated by clinical examples in this report, support the above mentioned observation. The timely, uncomplicated and biological techniques for uncovering of palatally impacted canines are of utmost importance for the successful treatment ot this condition.

\section{REFERENCES:}

1. Moyers RE: Handbook of Orthodontics 4th ed. Chicago,IL, Year book Medical Publisher 1988.

2. Becker A., Smith P, Behar R. The incidence of anomalous maxillary lateral incisors in relation to palatally-displaced cuspids. Angle Orthod. 1981 Jan;51(1):2429. [PubMed]

3. Ericson S, Kurol J. Radiographic examination of ectopically erupting maxillary canines. Am J Orthod Dentofacial Orthop 1987 Jun;91(6):483-492. doi: 10.1016/0889-5406(87)90005-9 CrossRef] [PubMed]

4. Ericson S, Kurol J. Early treatment of palatally erupting maxillary canines by extraction of the primary canines. Eur J Orthod 1988 Nov;10(4):283-245. [PubMed]

5. Bedoya M., Park JH. A review of the diagnosis and management of impacted maxillary canines. J Am Dent Assoc. 2009 Dec;140(12):1485-1493. [PubMed]

6. Kokich VG Preorthodontic uncovering and autonomous eruption of palatally impacted maxillary canines. Semin Orthod. 2010;16: 205-211.

7. Parkin N, Benson PE, Thind B, Shah A. Open versus closed surgical exposure of canine teeth that are displaced in the roof of the mouth. Cochrane Database Syst Rev. 2008 Oct 8;(4):CD006966. DOI: 10.1002/ 14651858.CD006966.pub2 CrossRef] [PubMed]

8. Swart RJ., Kiekens RM, Berge SJ, Kuijpers-Jagtman AM. Orthodontics in general practice. 2. Treatment of eruption failures. Ned Tijdschr Tandheelkd. 2007 Oct; 114(10): 416-22. [Article in Dutch] [PubMed]

9. Shafi I. No evidence to support one surgical technique over the other for the management of palatally displaced canines. Evid Based Dent. 2008;9(4):111. doi:10.1038/sj.ebd.6400613 CrossRef] [PubMed]

10. Schmidt AD, Kokich VG. Periodontal response to early uncovering, autonomous eruption and orthodontic alignment of palatally impacted maxillary canines. Am J Orthod Dentofacial Orthop 2007 Apr;131(4):449-455 doi:10.1016/ j.ajodo.2006.04.028 CrossRef] [PubMed]

11. Frank CA., Long M. Periodontal concerns associated with the orthodontic treatment of impacted teeth. Am J Orthod Dentofacial Orthop 2002 Jun;121(6): 63949. doi:10.1067/mod.2002.122396 CrossRef] [PubMed]

12. Kokich VG, Mathew DS. Impacted teeth: Orthodontic and surgical conciderations. In: McNamara Jr, editor. Orthodontics and dentofacial orthopedics. Ann Arbor (Mich): Needham Press; 2001: 395-422.

13. Ngan P, Hornbrook R., Weaver B. Early timely management of ectopically erupting maxillary canines. Semin Orthod 2005; 11: 152-163.

14. Frank Ch A. Treatment options for impacted teeth. J Am Dent Assoc. 2000 May;131(5):623-632. [PubMed]

\footnotetext{
Address for correspondence:

Dr. Miroslava Yordanova

Department of Orthodontics, Faculty of Dentistry, Medical University, Plovdiv

2, Hristo Botev Str., 4000 Plovdiv, Bulgaria

Mobile: +359898203355

E-mail:mirajord@gmail.com
} 